\title{
A DISCRETE WEIGHTED MONTGOMERY IDENTITY AND OSTROWSKI TYPE INEQUALITIES FOR FUNCTIONS OF TWO VARIABLES
}

\author{
A. AGLIĆ ALJINOVIĆ AND J. PEČARIĆ
}

\begin{abstract}
A discrete analogue of the weighted Montgomery identity for functions of two variables is presented and used to obtain new discrete Ostrowski type inequalities.
\end{abstract}

\section{INTRODUCTION}

In [3] Ostrowski showed that

$$
\left|f(x)-\frac{1}{b-a} \int_{a}^{b} f(t) d t\right| \leq\left[\frac{1}{4}+\frac{\left(x-\frac{a+b}{2}\right)^{2}}{(b-a)^{2}}\right](b-a)\left\|f^{\prime}\right\|_{\infty},
$$

for every $x \in[a, b]$ whenever $f:[a, b] \rightarrow \mathbb{R}$ is continuous on $[a, b]$ and differentiable on $(a, b)$ with derivative $f^{\prime}:(a, b) \rightarrow \mathbb{R}$ bounded on $(a, b)$ i.e.

$$
\left\|f^{\prime}\right\|_{\infty}=\sup _{t \in(a, b)}\left|f^{\prime}(t)\right|<+\infty .
$$

The weighted Montgomery identity (Pečarić [7]) is

$$
f(x)=\int_{a}^{b} w(t) f(t) d t+\int_{a}^{b} P_{w}(x, t) f^{\prime}(t) d t,
$$

where $f:[a, b] \rightarrow \mathbb{R}$ is differentiable on $[a, b], f^{\prime}:[a, b] \rightarrow \mathbb{R}$ integrable on $[a, b]$ and $w:[a, b] \rightarrow[0, \infty\rangle$ is some normalized weight function, i.e. an integrable function satisfying $\int_{a}^{b} w(t) d t=1, W(t)=\int_{a}^{t} w(x) d x$ for $t \in[a, b]$. The weighted Peano kernel is

$$
P_{w}(x, t)=\left\{\begin{array}{cc}
W(t), & a \leq t \leq x, \\
W(t)-1 & x<t \leq b .
\end{array}\right.
$$

2010 Mathematics Subject Classification. 26D15.

Key words and phrases. Discrete Montgomery identity, discrete Ostrowski inequality. 
For the uniform weight function $w(t)=\frac{1}{b-a}, t \in[a, b]$ (1.1) reduces to the Montgomery identity (see for instance [6]),

$$
f(x)=\frac{1}{b-a} \int_{a}^{b} f(t) d t+\int_{a}^{b} P(x, t) f^{\prime}(t) d t,
$$

where

$$
P(x, t)= \begin{cases}\frac{t-a}{b-a}, & a \leq t \leq x, \\ \frac{t-b}{b-a}, & x<t \leq b .\end{cases}
$$

Note that Ostrowski's inequality can be formulated in the equivalent form

$$
\left|f(x)-\frac{1}{b-a} \int_{a}^{b} f(t) d t\right| \leq \frac{(x-a)^{2}+(b-x)^{2}}{2(b-a)}\left\|f^{\prime}\right\|_{\infty}
$$

which can easily be obtained using (1.2) since,

$$
\begin{aligned}
& \left|\int_{a}^{b} P(x, t) f^{\prime}(t) d t\right| \leq\left\|f^{\prime}\right\|_{\infty} \int_{a}^{b}|P(x, t)| d t \\
& \leq\left(\int_{a}^{x}\left|\frac{t-a}{b-a}\right| d t+\int_{x}^{b}\left|\frac{t-b}{b-a}\right| d t\right)\left\|f^{\prime}\right\|_{\infty}=\frac{(x-a)^{2}+(b-x)^{2}}{2(b-a)}\left\|f^{\prime}\right\|_{\infty} .
\end{aligned}
$$

In a similar manner various generalizations of the Montgomery identity can be used to obtain generalizations of the Ostrowski inequality.

In [8] Pečarić and Vukelić presented integral weighted Montgomery identities for functions of two variables and applied them to obtain new inequalities of the Ostrowski type for mappings of two independent variables of which Theorem 1, below, is an example.

Theorem 1. Let $q:[a, b] \rightarrow \mathbf{R}$ and $h:[c, d] \rightarrow \mathbf{R}$ be two integrable functions. Let also $f:[a, b] \times[c, d] \rightarrow \mathbf{R}$ have continuous partial derivatives $\frac{\partial f(s, t)}{\partial s}, \frac{\partial f(s, t)}{\partial t}$, and $\frac{\partial^{2} f(s, t)}{\partial s \partial t}$ on $[a, b] \times[c, d]$, then for all $(x, y) \in[a, b] \times[c, d]$,

$$
\begin{array}{r}
f(x, y) P_{a}(b) P_{c}(d)=\int_{a}^{b} \int_{c}^{d} q(s) h(t) f(s, t) d s d t+P_{c}(d) \int_{a}^{b} \hat{P}(s) \frac{\partial f(s, y)}{\partial s} d s \\
+P_{a}(b) \int_{c}^{d} \tilde{P}(t) \frac{\partial f(x, t)}{\partial t} d t-\int_{a}^{b} \int_{c}^{d} \hat{P}(s) \tilde{P}(t) \frac{\partial^{2} f(s, t)}{\partial s \partial t} d s d t, \quad \text { (1.3) }
\end{array}
$$

where

$$
\begin{aligned}
P_{a}(s)=\int_{a}^{s} q(r) d r, \bar{P}_{a}(s)=P_{a}(b) & -P_{a}(s), P_{c}(t) \\
& =\int_{c}^{t} h(v) d v, \bar{P}_{c}(t)=P_{c}(d)-P_{c}(t),
\end{aligned}
$$


and

$$
\hat{P}(s)=\left\{\begin{array}{ll}
P_{a}(s), & a \leq s \leq x \\
-\bar{P}_{a}(s), & x<s \leq b
\end{array} \quad, \quad \tilde{P}(t)=\left\{\begin{array}{ll}
P_{c}(t), & c \leq t \leq y \\
-\bar{P}_{c}(t), & y<t \leq d
\end{array} .\right.\right.
$$

The special case of these identities for uniform weight functions were obtained in [4] and [5].

A discrete version of the Montgomery identity was given in [9]. Its generalizations were given in [1], [2] and also were used therein to obtain discrete Ostrowski type inequalities.

The aim of this paper is to prove a discrete analogue of the weighted Montgomery identity for function of two variables (1.3) and use it to obtain some new discrete Ostrowski type inequalities.

In Section 2. the identity for functions of two independent variables is presented. It is a discrete analogue of the result of [8] and, at the same time, a generalization for functions of two variables of the discrete weighted Montgomery identity obtained in [9].

In Section 3. this identity is used to obtain generalizations of Ostrowski inequality which are discrete analogues of the results of [4], [5] and [8] as well as generalizations for functions of two variable of the some results from [1] and [2].

\section{A Discrete Weighted Montgomery identity For FunCtions of TWO VARIABLES}

Let $f: \mathbb{R} \rightarrow \mathbb{R}$ be any real-to-real function. The difference operator $\Delta$ is defined by

$$
\Delta f(x)=f(x+1)-f(x),
$$

which is the finite analogue of the derivative and if $f$ is any real-to-real function, so too is $\Delta f$.

Let $w_{1}, w_{2}, \ldots, w_{n}$ be a finite sequence of real numbers and for $1 \leq k \leq n$, with

$$
W_{k}=\sum_{i=1}^{k} w_{i}, \quad \overline{W_{k}}=\sum_{i=k+1}^{n} w_{i}=W_{n}-W_{k} .
$$

The discrete weighted Montgomery identity (see [9]) states that

$$
f(k)=\frac{1}{W_{n}} \sum_{i=1}^{n} w_{i} f(i)+\sum_{i=1}^{n} D_{w}(k, i) \Delta f(i),
$$

where the discrete weighted Peano kernel is defined by

$$
D_{w}(k, i)=\frac{1}{W_{n}} \cdot\left\{\begin{array}{cc}
W_{i}, & 1 \leq i \leq k-1, \\
\left(-\overline{W_{i}}\right), & k \leq i \leq n .
\end{array}\right.
$$


For uniform weights $w_{i}=1, \quad i=1, \ldots, n$, and (2.2) reduces to the discrete Montgomery identity

$$
f(k)=\frac{1}{n} \sum_{i=1}^{n} f(i)+\sum_{i=1}^{n} D(k, i) \Delta f(i),
$$

where

$$
D(k, i)=\left\{\begin{array}{cc}
\frac{i}{n}, & 1 \leq i \leq k-1, \\
\frac{i}{n}-1, & k \leq i \leq n .
\end{array}\right.
$$

Remark 1. More generally, for any $x \in \mathbb{R}$ it holds that

$$
f(x+k)=\frac{1}{W_{n}} \sum_{i=1}^{n} w_{i} f(x+i)+\sum_{i=1}^{n} D_{w}(k, i) \Delta f(x+i) .
$$

Without loss of generality, it will subsequently be assumed that $x=0$.

Definition 1. For $f: \mathbb{R}^{2} \rightarrow \mathbb{R}$, the difference operators $\boldsymbol{\Delta}_{1}$ and $\boldsymbol{\Delta}_{2}$ are defined as

$$
\begin{aligned}
& \Delta_{1} f(i, j)=f(i+1, j)-f(i, j), \\
& \Delta_{2} f(i, j)=f(i, j+1)-f(i, j),
\end{aligned}
$$

these are the finite analogue of the first partial derivatives.

Theorem 2. Let $f: \mathbb{R}^{2} \rightarrow \mathbb{R}$ be any real function of two variables, $n, m \in \mathbb{N}$ and $w_{1}, w_{2}, \ldots, w_{n}$ and $\varphi_{1}, \varphi_{2}, \ldots, \varphi_{m}$ finite sequences of positive real numbers. Further, let $W_{k}=\sum_{i=1}^{k} w_{i}, \overline{W_{k}}=W_{n}-W_{k}$ for $1 \leq k \leq n$ and $\Phi_{l}=\sum_{j=1}^{l} \varphi_{j}, \overline{\Phi_{l}}=\Phi_{m}-\Phi_{l}$ for $1 \leq l \leq m$, then,

$$
\begin{gathered}
f(k, l)=\frac{1}{W_{n} \Phi_{m}} \sum_{i=1}^{n} \sum_{j=1}^{m} w_{i} \varphi_{j} f(i, l)+\frac{1}{W_{n}} \sum_{i=1}^{n} \sum_{j=1}^{m} w_{i} D_{\varphi}(l, j) \Delta_{2} f(i, j) \\
+\frac{1}{\Phi_{m}} \sum_{i=1}^{n} \sum_{j=1}^{m} \varphi_{j} D_{w}(k, i) \Delta_{1} f(i, j)+\sum_{i=1}^{n} \sum_{j=1}^{m} D_{w}(k, i) D_{\varphi}(l, j) \Delta_{1} \Delta_{2} f(i, j)
\end{gathered}
$$

where

$$
\begin{aligned}
& D_{w}(k, i)=\frac{1}{W_{n}} \cdot\left\{\begin{array}{cc}
W_{i}, & 1 \leq i \leq k-1, \\
\left(-\overline{W_{i}}\right), & k \leq i \leq n,
\end{array}\right. \\
& D_{\varphi}(l, j)=\frac{1}{\Phi_{m}} \cdot\left\{\begin{array}{cc}
\Phi_{j}, & 1 \leq j \leq l-1, \\
\left(-\overline{\Phi_{j}}\right), & l \leq j \leq m .
\end{array}\right.
\end{aligned}
$$


Proof. Using (2.2) on the first variable, we have

$$
f(k, l)=\frac{1}{W_{n}} \sum_{i=1}^{n} w_{i} f(i, l)+\sum_{i=1}^{n} D_{w}(k, i) \Delta_{1} f(i, l)
$$

and further, with regard to the second variable

$$
f(i, l)=\frac{1}{\Phi_{m}} \sum_{j=1}^{m} \varphi_{j} f(i, j)+\sum_{j=1}^{m} D_{\varphi}(l, j) \Delta_{2} f(i, j) .
$$

Applying $\Delta_{1}$ on the latter identity gives

$$
\Delta_{1} f(i, l)=\frac{1}{\Phi_{m}} \sum_{j=1}^{m} \varphi_{j} \Delta_{1} f(i, j)+\sum_{j=1}^{m} D_{\varphi}(l, j) \Delta_{1} \Delta_{2} f(i, j) .
$$

Substituting the last two identities into the first leads to (2.4).

For the special case of uniform weights, we obtain the following generalization of (2.3) for functions of two independent variables.

Corollary 1. Let $f: \mathbb{R}^{2} \rightarrow \mathbb{R}$ be any real function of two variables, $n, m, k, l$ $\in \mathbb{N}, k \leq n, l \leq m$. Then it holds

$$
\begin{aligned}
& f(k, l)=\frac{1}{n m} \sum_{i=1}^{n} \sum_{j=1}^{m} f(i, l)+\frac{1}{n} \sum_{i=1}^{n} \sum_{j=1}^{m} D_{2}(l, j) \Delta_{2} f(i, j) \\
& +\frac{1}{m} \sum_{i=1}^{n} \sum_{j=1}^{m} D_{1}(k, i) \Delta_{1} f(i, j)+\sum_{i=1}^{n} \sum_{j=1}^{m} D_{1}(k, i) D_{2}(l, j) \Delta_{1} \Delta_{2} f(i, j)
\end{aligned}
$$

where

$$
\begin{aligned}
& D_{1}(k, i)=\left\{\begin{array}{cc}
\frac{i}{n}, & 1 \leq i \leq k-1, \\
\frac{i}{n}-1, & k \leq i \leq n,
\end{array}\right. \\
& D_{2}(l, j)=\left\{\begin{array}{cc}
\frac{j}{m}, & 1 \leq j \leq l-1, \\
\frac{j}{m}-1, & l \leq j \leq m .
\end{array}\right.
\end{aligned}
$$

Proof. If we take $w_{i}=1, i=1, \ldots, n$ and $\varphi_{j}=1, j=1, \ldots, m$, we have $W_{i}=i, \overline{W_{i}}=n-i$ and $\Phi_{j}=j, \overline{\Phi_{j}}=m-j$. In this case (2.4) reduces to $(2.5)$.

\section{Discrete Ostrowski type inequalities}

Here we derive new Ostrowski type inequalities for functions $f$ of two variables. 
Definition 2. We say $(p, q)$ is a pair of conjugate exponents if $1<p, q<\infty$ and $\frac{1}{p}+\frac{1}{q}=1$; or if $p=1$ and $q=\infty$; or if $p=\infty$ and $q=1$.

Theorem 3. Suppose that all the assumptions of Theorem 2 hold. Additionally assume that $(p, q)$ is a pair of conjugate exponents, $1 \leq p, q \leq \infty$, then,

$$
\begin{gathered}
\mid f(k, l)-\frac{1}{W_{n} \Phi_{m}} \sum_{i=1}^{n} \sum_{j=1}^{m} w_{i} \varphi_{j} f(i, l)-\frac{1}{W_{n}} \sum_{i=1}^{n} \sum_{j=1}^{m} w_{i} D_{\varphi}(l, j) \Delta_{2} f(i, j) \\
-\frac{1}{\Phi_{m}} \sum_{i=1}^{n} \sum_{j=1}^{m} \varphi_{j} D_{w}(k, i) \Delta_{1} f(i, j) \mid \leq\left\|D_{w}(k, \cdot)\right\|_{q}\left\|D_{\varphi}(l, \cdot)\right\|_{q}\left\|\Delta_{1} \Delta_{2} f\right\|_{p}
\end{gathered}
$$

where

$$
\|g\|_{p}=\left\{\begin{array}{c}
\left(\sum_{i}|g(i)|^{p}\right)^{\frac{1}{p}}, \quad \text { if } 1 \leq p<\infty \\
\max _{i}|g(i)|, \quad \text { if } p=\infty
\end{array}\right.
$$

if $g$ is function of one variable and

$$
\|g\|_{p}=\left\{\begin{array}{cc}
\left(\sum_{i, j}|g(i, j)|^{p}\right)^{\frac{1}{p}}, & \text { if } 1 \leq p<\infty \\
\max _{i, j}|g(i, j)|, & \text { if } p=\infty
\end{array}\right.
$$

if $g$ is function of two variables.

Proof. By using (2.4)

$$
\begin{array}{r}
\mid f(k, l)-\frac{1}{W_{n} \Phi_{m}} \sum_{i=1}^{n} \sum_{j=1}^{m} w_{i} \varphi_{j} f(i, l)-\frac{1}{W_{n}} \sum_{i=1}^{n} \sum_{j=1}^{m} w_{i} D_{\varphi}(l, j) \Delta_{2} f(i, j) \\
-\frac{1}{\Phi_{m}} \sum_{i=1}^{n} \sum_{j=1}^{m} \varphi_{j} D_{w}(k, i) \Delta_{1} f(i, j) \mid \\
=\left|\sum_{i=1}^{n} \sum_{j=1}^{m} D_{w}(k, i) D_{\varphi}(l, j) \Delta_{1} \Delta_{2} f(i, j)\right|
\end{array}
$$

and applying the discrete Hölder inequality for double sums

$$
\left|\sum_{i=1}^{n} \sum_{j=1}^{m} D_{w}(k, i) D_{\varphi}(l, j) \Delta_{1} \Delta_{2} f(i, j)\right|
$$




$$
\begin{aligned}
& \leq\left(\sum_{i=1}^{n} \sum_{j=1}^{m}\left|D_{w}(k, i) D_{\varphi}(l, j)\right|^{q}\right)^{\frac{1}{q}} \cdot\left(\sum_{i=1}^{n} \sum_{j=1}^{m}\left|\Delta_{1} \Delta_{2} f(i, j)\right|^{p}\right)^{\frac{1}{p}} \\
& =\left(\sum_{i=1}^{n}\left|D_{w}(k, i)\right|^{q}\right)^{\frac{1}{q}}\left(\sum_{j=1}^{m}\left|D_{\varphi}(l, j)\right|^{q}\right)^{\frac{1}{q}}\left(\sum_{i=1}^{n} \sum_{j=1}^{m}\left|\Delta_{1} \Delta_{2} f(i, j)\right|^{p}\right)^{\frac{1}{p}}
\end{aligned}
$$

we obtain (3.1).

In the next three corollaries we give the special cases for uniform weights. First, for $n \in \mathbb{N}$ and $m \in \mathbb{R}$ we denote by $S_{m}(n)$, the sum

$$
1^{m}+2^{m}+3^{m}+\cdots+(n-1)^{m} .
$$

It is well known that if $m \in \mathbb{N}$ (see [10])

$$
S_{m}(n)=\frac{1}{m+1} \sum_{i=0}^{m}\left(\begin{array}{c}
m+1 \\
i
\end{array}\right) B_{i} n^{m+1-i}
$$

where $B_{i}, i \geq 0$ are Bernoulli numbers, defined by,

$$
\sum_{i=0}^{m}\left(\begin{array}{c}
m+1 \\
i
\end{array}\right) B_{i}= \begin{cases}1, & \text { if } m=0 \\
0, & \text { if } m \neq 0 .\end{cases}
$$

Corollary 2. Let $f: \mathbb{R}^{2} \rightarrow \mathbb{R}$ be any real function of two variables, $n, m, k, l$ $\in \mathbb{N}, k \leq n, l \leq m$, then

$$
\begin{aligned}
& \mid f(k, l)-\frac{1}{n m} \sum_{i=1}^{n} \sum_{j=1}^{m} f(i, l) \\
& \quad-\frac{1}{n} \sum_{i=1}^{n} \sum_{j=1}^{m} D_{2}(l, j) \Delta_{2} f(i, j)-\frac{1}{m} \sum_{i=1}^{n} \sum_{j=1}^{m} D_{1}(k, i) \Delta_{1} f(i, j) \mid \\
& \leq \frac{1}{n m}\left(\frac{n^{2}-1}{4}+\left(k-\frac{n+1}{2}\right)^{2}\right)\left(\frac{m^{2}-1}{4}+\left(l-\frac{m+1}{2}\right)^{2}\right) \cdot\left\|\Delta_{1} \Delta_{2} f\right\|_{\infty} .
\end{aligned}
$$

Proof. We take $w_{i}=1, i=1, \ldots, n ; \varphi_{j}=1, j=1, \ldots, m$, and $p=\infty$ in (3.1) for which we have $W_{i}=i, \overline{W_{i}}=n-i, \Phi_{j}=j, \overline{\Phi_{j}}=m-j$, giving,

$$
\begin{aligned}
\left\|D_{w}(k, \cdot)\right\|_{1} & =\sum_{i=1}^{n}\left|D_{1}(k, i)\right|=\sum_{i=1}^{k-1} \frac{i}{n}+\sum_{i=k}^{n}\left(1-\frac{i}{n}\right) \\
& =\frac{1}{n}\left(\frac{k(k-1)}{2}+\frac{(n-k+1)(n-k)}{2}\right) \\
& =\frac{1}{n}\left(\frac{n^{2}-1}{4}+\left(k-\frac{n+1}{2}\right)^{2}\right)
\end{aligned}
$$


and similarly

$$
\begin{aligned}
\left\|D_{\varphi}(l, \cdot)\right\|_{1} & =\sum_{j=1}^{m}\left|D_{2}(l, j)\right|=\sum_{j=1}^{l-1} \frac{j}{m}+\sum_{i=k}^{m}\left(1-\frac{j}{m}\right) \\
& =\frac{1}{m}\left(\frac{m^{2}-1}{4}+\left(l-\frac{m+1}{2}\right)^{2}\right) .
\end{aligned}
$$

Corollary 3. Let $f: \mathbb{R}^{2} \rightarrow \mathbb{R}$ be any real function of two variables, $n, m, k, l$ $\in \mathbb{N}, k \leq n, l \leq m$, then

$$
\begin{aligned}
\mid f(k, l)-\frac{1}{n m} \sum_{i=1}^{n} \sum_{j=1}^{m} f(i, l)-\frac{1}{n} \sum_{i=1}^{n} \sum_{j=1}^{m} D_{2}(l, j) \Delta_{2} f(i, j) \\
\quad-\frac{1}{m} \sum_{i=1}^{n} \sum_{j=1}^{m} D_{1}(k, i) \Delta_{1} f(i, j) \mid \\
\leq \frac{1}{n m} \max \{k-1, n-k\} \cdot \max \{l-1, m-l\} \cdot\left\|\Delta_{1} \Delta_{2} f\right\|_{1} .
\end{aligned}
$$

Proof. We take $w_{i}=1, \quad i=1, \ldots, n ; \quad \varphi_{j}=1, j=1, \ldots, m$ and $p=1$ in (3.1), with again, $W_{i}=i, \overline{W_{i}}=n-i, \Phi_{j}=j, \overline{\Phi_{j}}=m-j$, giving,

$$
\left\|D_{w}(k, \cdot)\right\|_{\infty}=\max _{1 \leq i \leq n}\left\{\left|D_{1}(k, i)\right|\right\}=\frac{1}{n} \max \{k-1, n-k\}
$$

and

$$
\left\|D_{\varphi}(l, \cdot)\right\|_{\infty}=\max _{1 \leq j \leq m}\left\{\left|D_{2}(l, j)\right|\right\}=\frac{1}{m} \max \{l-1, m-l\} .
$$

Corollary 4. Let $f: \mathbb{R}^{2} \rightarrow \mathbb{R}$ be any real function of two variables, $n, m, k, l$ $\in \mathbb{N}, k \leq n, l \leq m$. Let also $(p, q)$ be a pair of conjugate exponents, $1<p, q<\infty$, then

$$
\begin{aligned}
& \mid f(k, l)-\frac{1}{n m} \sum_{i=1}^{n} \sum_{j=1}^{m} f(i, l)-\frac{1}{n} \sum_{i=1}^{n} \sum_{j=1}^{m} D_{2}(l, j) \Delta_{2} f(i, j) \\
& \quad-\frac{1}{m} \sum_{i=1}^{n} \sum_{j=1}^{m} D_{1}(k, i) \Delta_{1} f(i, j) \mid \\
& \leq \frac{1}{n m}\left[\left(S_{q}(k)+S_{q}(n-k+1)\right)\left(S_{q}(l)+S_{q}(m-l+1)\right)\right]^{\frac{1}{q}} \cdot\left\|\Delta_{1} \Delta_{2} f\right\|_{p} .
\end{aligned}
$$


Proof. We take $w_{i}=1, \quad i=1, \ldots, n$ and $\varphi_{j}=1, \quad j=1, \ldots, m$ in (3.1), $W_{i}=i, \overline{W_{i}}=n-i, \Phi_{j}=j, \overline{\Phi_{j}}=m-j$, giving,

$$
\begin{aligned}
\left\|D_{w}(k, \cdot)\right\|_{q} & =\left(\sum_{i=1}^{n}\left|D_{1}(k, i)\right|^{q}\right)^{\frac{1}{q}}=\frac{1}{n}\left(\sum_{i=1}^{k-1} i^{q}+\sum_{i=k}^{n}(n-i)^{q}\right)^{\frac{1}{q}} \\
& =\frac{1}{n}\left(S_{q}(k)+S_{q}(n-k+1)\right)^{\frac{1}{q}}
\end{aligned}
$$

and similarly

$$
\begin{aligned}
\left\|D_{\varphi}(l, \cdot)\right\|_{q} & =\left(\sum_{j=1}^{m}\left|D_{2}(l, j)\right|^{q}\right)^{\frac{1}{q}}=\frac{1}{m}\left(\sum_{j=1}^{l-1} j^{q}+\sum_{i=l}^{m}(m-j)^{q}\right)^{\frac{1}{q}} \\
& =\frac{1}{m}\left(S_{q}(l)+S_{q}(m-l+1)\right)^{\frac{1}{q}} .
\end{aligned}
$$

Remark 2. Taking $q \in \mathbb{N}$ in (3.2) is equivalent to having

$$
\begin{aligned}
& \mid f(k, l)-\frac{1}{n m} \sum_{i=1}^{n} \sum_{j=1}^{m} f(i, l) \\
& -\frac{1}{n} \sum_{i=1}^{n} \sum_{j=1}^{m} D_{2}(l, j) \Delta_{2} f(i, j)-\frac{1}{m} \sum_{i=1}^{n} \sum_{j=1}^{m} D_{1}(k, i) \Delta_{1} f(i, j) \mid \\
& \leq \frac{1}{n m}\left(\frac{1}{q+1} \sum_{i=0}^{q}\left(\begin{array}{c}
q+1 \\
i
\end{array}\right) B_{i}\left(k^{q+1-i}+(n-k+1)^{q+1-i}\right)\right)^{\frac{1}{q}} \\
& \cdot\left(\frac{1}{q+1} \sum_{i=0}^{q}\left(\begin{array}{c}
q+1 \\
i
\end{array}\right) B_{i}\left(l^{q+1-i}+(m-l+1)^{q+1-i}\right)\right)^{\frac{1}{q}} \cdot\left\|\Delta_{1} \Delta_{2} f\right\|_{p},
\end{aligned}
$$

where $B_{i}, i \geq 0$ are Bernoulli numbers.

Acknowledgement. This research was supported by the Croatian Ministry of Science, Education, and Sports, under Research Grants 036-1170889-1054 (first author) and 117-1170889-0888 (second author).

\section{REFERENCES}

[1] A. Aglić Aljinović, J. Pečarić, Discrete weighted Montgomery identity and discrete Ostrowski type inequalities, Comp. Math. Appl., 48 (2004), 731-745.

[2] A. Aglić Aljinović, J. Pečarić, A discrete Euler identity, JIPAM, 5 (3) (2004), article 58. 
[3] A. Ostrowski, Über die Absolutabweichung einer differentiebaren Funktion von ihrem Integralmittelwert, Comment. Math. Helv., 10 (1938), 226-227.

[4] N. S. Barnett, S. S. Dragomir, An Ostrowski type inequality for double integrals and applications for cubature formulae, Soochow J. of Math., 27 (1) (2001), 1-10.

[5] S. S. Dragomir, N. S. Barnett, P. Cerone; An Ostrowski type inequality for double integrals in terms of $L_{p}$-norms and applications in numerical integration, Revue D'analyse Numerique et de Theorie De L'Approximation, 32 (2) (2003), 161-169.

[6] D. S. Mitrinović, J. E. Pečarić, and A. M. Fink, Inequalities for Functions and their Integrals and Derivatives, Kluwer Academic Publishers, Dordrecht, 1994.

[7] J. Pečarić, On the Čebyšev inequality, Bul. Inst. Politehn. Timisoara, 25 (39) (1980), $10-11$.

[8] J. Pečarić, I. Perić, A. Vukelić, Montgomery's identities for function of two variables, JMAA, 332 (1) (2007), 617-630.

[9] D. S. Mitrinović. J. E. Pečarić, Monotone funkcije i nijhove nejednakosti, Naučna kniga, Beograd 1990.

[10] Graham, Knuth, Patashnik, Concrete Mathematics: A Foundation for Computer Science, Second edition, Addison-Wesley 1994.

(Received: February 14, 2012)

(Revised: May 17, 2012)

\author{
A. Aglić Aljinović \\ Department of applied mathematics \\ Faculty of Electrical Engineering and Computing \\ Unska 3, 10000 Zagreb \\ Croatia \\ E-mail: andrea.aglic@fer.hr \\ J. Pečarić \\ Faculty of Textile technology \\ University of Zagreb \\ Prilaz baruna Filipovića 28a, 10000 Zagreb \\ Croatia \\ E-mail: pecaric@hazu.hr
}

